\title{
Behavioral and Neural Effects of Nicotine on Visuospatial Attentional Reorienting in Non-Smoking Subjects
}

\author{
Simone Vossel*, 1,2 , Christiane M Thiel ${ }^{1,2,3}$ and Gereon R Fink ${ }^{1,2,4}$ \\ 'Department of Medicine, Institute of Neuroscience and Biophysics, Research Centre Jülich, Jülich, Germany; ${ }^{2}$ Brain Imaging Centre West, \\ Research Centre Jülich, Jülich, Germany; ${ }^{3}$ Institute of Biology and Environmental Sciences, University of Oldenburg, Oldenburg, Germany; \\ ${ }^{4}$ Department of Neurology, University Hospital Cologne, Cologne, Germany
}

\begin{abstract}
The cholinergic neurotransmitter system has been proposed to be involved in the processing of probabilistic top-down information provided by endogenous cues in location-cueing paradigms. It has been shown that the behavioral and neural effects of a nicotinic cholinergic stimulation resemble the effects obtained by manipulating the validity of the spatial cues: enhancing cortical nicotine levels and decreasing cue validity both reduce the reaction time difference between invalidly and validly cued targets (ie, the 'validity effect') as well as neural activity related to attentional reorienting in parietal brain regions. In the present study, we investigated whether the behavioral and neural effects of nicotine in location-cueing paradigms are dependent upon different a priori cue validities. Twenty-four subjects were investigated in a double-blind placebo-controlled between-subject design with functional magnetic resonance imaging. Nicotine was administered to non-smoking volunteers via polacrilex gums (Nicorette ${ }^{\mathbb{R}}, 2 \mathrm{mg}$ ) before performing a location-cueing paradigm with valid and invalid cues in the context of 90 and $60 \%$ cue validity in the MR scanner. Nicotine significantly reduced the validity effect in the $90 \%$ but not in the $60 \%$ cue validity condition. Fronto-parietal and cingulate regions showed stronger nicotinic reductions of reorientingrelated neural activity in the high than in the low cue validity condition. Our data reveal an interaction effect between the pharmacological and cognitive modulation of attentional reorienting, which is evident at both a behavioral as well as the neuronal level.

Neuropsychopharmacology (2008) 33, 731-738; doi:I0.1038/sj.npp. I301469; published online 6 June 2007
\end{abstract}

Keywords: neuropharmacology; acetylcholine; $\mathrm{AMRl}$; selective attention; location-cueing paradigm; cue validity

\section{INTRODUCTION}

The cholinergic agonist nicotine has been shown to improve a variety of attentional processes in smoking and nonsmoking human subjects (for a review, see, eg, Newhouse et al, 2004 or Rezvani and Levin, 2001). One attentional function that is regarded to be mediated by cholinergic neurotransmission is reorienting of visuospatial attention (Posner and Fan, 2004). Attentional reorienting can be investigated in location-cueing paradigms when misleading advance information is provided by a spatially invalid cue. The difference in reaction times (RTs) to invalidly and validly cued targets is termed the 'validity effect' and is used as a behavioral measure for attentional reorienting. Human and animal evidence suggest that nicotine improves reorienting of attention, as it was shown that nicotinic cholinergic stimulation reduces RTs to invalidly cued targets resulting in a reduction of the validity effect (Witte et al, 1997; Murphy and Klein, 1998; Thiel et al, 2005;

*Correspondence: S Vossel, Department of Medicine, Institute of Neuroscience and Biophysics, Research Centre Jülich, Leo-BrandtStreet 5, 52425 Jülich, Germany, Tel: + 492461 6I 4007, Fax: + 49 246 | 61 2820, E-mail: s.vossel@fz-juelich.de

Received 7 February 2007; revised 6 May 2007; accepted 8 May 2007
Phillips et al, 2000; Stewart et al, 2001; see, however, Griesar et al, 2002).

$\mathrm{Yu}$ and Dayan (2005) recently proposed that the neurotransmitter acetylcholine signals 'expected uncertainty', that is, the degree of unreliability of predictive relationships. Their model suggests that the reduction of the validity effect under nicotine is due to reduced reliance on top-down information provided by the cue. In other words, it is proposed that nicotine reduces the degree to which the spatial cue can be trusted and thus suppresses the use of the cue for attentional allocation. Thus, the behavioral effect of nicotine in location-cueing paradigms resembles the effect obtained by manipulating cue validity (ie, the ratio between valid and invalid trials): prior work has shown that decreasing cue validity increases RTs to validly cued targets and decreases RTs to invalidly cued targets (Jonides, 1980; Eriksen and Yeh, 1985; Madden, 1992; Riggio and Kirsner, 1997). Interestingly, this cognitive modulation of attentional reorienting has been observed in studies using peripheral cue stimuli, which are thought to elicit automatic (exogenous) attentional orienting (Eriksen and Yeh, 1985; Madden, 1992) as well as centrally presented cue stimuli inducing voluntary (endogenous) attention shifts (Jonides, 1980; Riggio and Kirsner, 1997; Vossel et al, 2006). Regarding the pharmacological modulation (ie, the nicotine-induced 
reduction of the validity effect), existing animal and human studies have employed central predictive (Thiel et al, 2005), peripheral predictive (Witte et al, 1997; Murphy and Klein, 1998; Stewart et al, 2001), as well as non-predictive cues (50\% cue validity; Phillips et al, 2000). However, with regard to human subjects, it has been shown that nicotine exerts its effect in paradigms with central predictive but not with peripheral non-predictive cueing (Meinke et al, 2006) suggesting that the top-down information about the cue-target relationship may play an important role in the pharmacological effect. One could speculate that findings from animal studies are thus not perfectly transferable to human research and with regard to the effects of cue validity manipulations on RTs divergent effects between humans and animals have indeed been reported (Bowman et al, 1993).

We have previously shown that both the manipulation of cholinergic neurotransmission and the manipulation of cue validity modulate reorienting-related brain activity in parietal and temporo-parietal areas (Thiel et al, 2005; Vossel et al, 2006). In particular, in these regions, both nicotinic stimulation and low cue validity reduce neural activity related to attentional reorienting. The present study aims at investigating the nicotinic modulation of the validity effect in conditions of high and low cue validity. We employed a location-cueing paradigm with valid and invalid trials in the context of high and low cue validity (90 and 60\%) and investigated reorienting-related neural activity under placebo and nicotine. We hypothesized that the modulatory effects of nicotine should depend on the a priori validity of the spatial cue with nicotine reducing the validity effect particularly in the context of high cue validity.

\section{MATERIALS AND METHODS}

\section{Subjects}

The study was carried out in accordance with the ethical principles of the World Medical Association (Declaration of Helsinki). Twenty-six subjects gave written informed consent to participate in the study. All were right-handed as indexed by a handedness inventory (Oldfield, 1971) and had no history of neurological or psychiatric disease. To avoid confounding effects with withdrawal from nicotine all subjects had to be non-smoking since at least 2 years. No subject was on medication (except for contraceptives). Two subjects were excluded from further analysis due to excessive head movement $(>3 \mathrm{~mm})$ during functional magnetic resonance imaging (fMRI) scanning. Therefore, data from 24 subjects were analyzed. To avoid confounding effects owing to repeated measurements (Hills and Armitage, 1979; Millar, 1983), we used a between-subject design in which the subjects were randomly assigned to the nicotine or placebo group, respectively (placebo group: six male subjects, six female subjects; mean age 25.7 years; nicotine group: seven male subjects, five female subjects; mean age 24.3 years). The data of the placebo group has been reported separately (Vossel et al, 2006).

\section{Drug Administration}

Drug administration was double-blinded. The subjects received either a nicotine polacrilex gum (Nicorette ${ }^{\circledR}$
$2 \mathrm{mg}$, Pharmacia/Pfizer) or a placebo gum with matched taste (Pharmacia/Pfizer) and chewed it for $30 \mathrm{~min}$. They were instructed to chew once every $3 \mathrm{~s}$. Pulse rate was assessed and blood samples were taken approximately $25 \mathrm{~min}$ after drug and placebo administration. Nicotine blood serum levels were determined after liquid-liquid extraction using an isocratic high performance liquid chromatography with a reversed phase microbore column followed by UV detection. Subjective drug effects were assessed $20 \mathrm{~min}$ after drug administration with visual analogue scales for the three factors 'alertness', 'contentedness', and 'calmness' (Bond and Lader, 1974). Moreover, the subjects completed a symptom checklist asking for known negative side effects of nicotine.

\section{Stimuli and Experimental Paradigm}

We employed a location-cueing task with central predictive cues (Posner, 1980). Stimuli were projected onto a screen in front of the participant in the MR scanner with a viewing distance of approximately $29 \mathrm{~cm}$. Subjects were presented with two horizontally arranged boxes $\left(4.9^{\circ}\right.$ wide and $13.9^{\circ}$ eccentric in each visual field). A central diamond $\left(2.5^{\circ}\right.$ eccentric in each visual field) was placed in between serving as a fixation point. Cues consisted of a colored $100 \mathrm{~ms}$ brightening of one side of the diamond depicting an arrowhead pointing to one of the peripheral boxes.

There were two differently colored (green and blue) spatial cues that predicted the occurrence of the target with different validity (90 and $60 \%$, respectively). The assignment of cue color (green or blue) and cue validity (90 or $60 \%$ ) was counterbalanced across subjects. After a variable cue-target interval of 400 or $700 \mathrm{~ms}$, the cue was followed by the presentation of the target appearing for $100 \mathrm{~ms}$ in one of the two lateral boxes. Subjects were instructed to respond as quickly as possible to the target by a button press with the index finger of their right hand. Trials were presented randomly every $1800 \mathrm{~ms}$. One-third of the trials were 'null events' (Josephs and Henson, 1999) where a baseline stimulus was displayed, leading effectively to variable stimulus onset asynchronies (ie, 1800, 3600, $5400 \mathrm{~ms}$, so on). In addition to validly and invalidly cued trials, we included catch trials in which the cue was not followed by any target. The experiment consisted of 756 trials including 252 null events and lasted for $24 \mathrm{~min}$. To prevent deterioration in fixation ability, the scanning session included two rest periods of approximately $1 \mathrm{~min}$ during which the word 'pause' was shown on the display and the subjects were allowed to close their eyes. Before performing the task in the MR scanner, the subjects were informed about the different cue validities and completed a practice session of $8 \mathrm{~min}$.

\section{Data Acquisition}

T2*-weighted echoplanar (EPI) images with blood oxygen level-dependent (BOLD) contrast (matrix size $64 \times 64$, pixel size $3.12 \times 3.12 \times 5 \mathrm{~mm}^{3}$ ) were obtained using a $1.5 \mathrm{~T}$ Sonata MRI System (Siemens, Erlangen, Germany). Seven hundred forty-five volumes of twenty-one $4 \mathrm{~mm}$ thick axial slices were acquired sequentially with a $1.0 \mathrm{~mm}$ gap (repetition time $2.0 \mathrm{~s}$, echo time $60 \mathrm{~ms}$ ). The first five volumes were 
discarded to allow for T1 equilibration effects and were thus excluded from further data processing. To correct for interscan movement, the images were spatially realigned to the first of the remaining 740 volumes. The images were synchronized to the middle slice correcting for differences in slice acquisition time and normalized to a standard EPI template volume (resampled to $3 \times 3 \times 3 \mathrm{~mm}^{3}$ voxels). The data were smoothed with a Gaussian kernel of $8 \mathrm{~mm}$ fullwidth half-maximum to accommodate intersubject anatomical variability.

\section{Statistical Analysis Of Imaging Data}

Data were analyzed with Statistic Parametric Mapping software SPM2 (Wellcome Department of Imaging Neuroscience, London, Friston et al, 1995, http://www.fil. ion.ucl.ac.uk/spm2.html) employing a random effects model. For each subject, seven regressors were defined comprising four events of interest (validly cued targets in the context of $90 \%$ cue validity, invalidly cued targets in the context of $90 \%$ cue validity, validly cued targets in the context of $60 \%$ cue validity, invalidly cued targets in the context of $60 \%$ cue validity) and two events of no interest (catch trials, incorrect responses). The event types were time-locked to the onset of the target by a canonical synthetic hemodynamic response function (hrf). The pauses were modeled as blocks (convolved with the hrf) in a third regressor of no interest. The six movement parameters (rigid body translation in the $x$-, $y$ - and $z$-plane as well as rotation around the $x$-, $y$-, and $z$-axis) were included in the design matrix as additional regressors. For each subject, four contrast images were created (each trial type vs baseline). These first-level contrast images were entered into a mixed ANOVA model with the between-subject factor drug (placebo, nicotine). Inhomogeneity of variance and correlation of measurement were estimated with a Restricted Maximum Likelihood (ReML) algorithm. We focused our analysis on the effects of nicotine on reorienting-related neural activity dependent upon cue validity (ie, the three-way interaction of the factors cueing $\times$ cue validity $\times$ drug), and used the directed t-contrast (placebo ( $90 \%$ cue validity (invalid $>$ valid) $>60 \%$ cue validity (invalid $>$ valid) $)>$ nicotine $(90 \%$ cue validity (invalid $>$ valid) $>60 \%$ cue validity (invalid $>$ valid)) ) to isolate brain regions where nicotine reduces reorientingrelated brain activity to a greater extent in the high as compared with the low cue validity condition. Activations are reported at a level of $P<0.001$ uncorrected and a cluster threshold of more than three contiguous voxels and are shown on the mean image of the structural scans of all subjects. In addition, we correlated the parameter estimates (beta weights) for the voxel of peak activation in these regions with blood nicotine levels using Pearson's correlation coefficient.

\section{Statistical Analysis of Behavioral Data}

For RT analysis, we used a distributional analysis, fitting exGauss distributions to individual RT data (Heathcote et al, 2004). As RT distributions are usually not normally distributed but positively skewed (Heathcote et al, 1991), this procedure allows a more comprehensive analysis of RT data and therefore provides a more detailed characterization of pharmacological effects than traditional RT measures, enabling, for example, the analysis of RT variability. Distributional RT analyses have already been applied in prior studies using location-cueing paradigms (Gottlob, 2004; Vossel et al, 2006). RTs faster than $100 \mathrm{~ms}$ (ie, anticipations) were excluded from the analyses. Ex-Gauss functions consist of a Gaussian component (with the mean $\mu$ and the standard deviation $\sigma$ ) and an exponential component $\tau$. Probability density functions were plotted for the four experimental conditions according to the following formula:

$$
f(R T)=\frac{\mathrm{e}^{\left(\sigma^{2} / 2 \tau^{2}\right)-((R T-\mu) / \tau)}}{\tau \sqrt{2 \pi}} \int_{-\infty}^{((R T-\mu) / \sigma)-(\sigma / \tau)} \mathrm{e}^{-\left(y^{2} / 2\right)} \mathrm{d} y
$$

A $2 \times 2 \times 2$ mixed ANOVA model with the within-subject factors cueing (valid; invalid), cue validity (90;60\%), and the between-subject factor drug (placebo; nicotine) was calculated for the parameters $\mu, \sigma$, and $\tau$. Two-sample $t$-tests were used to compare the validity effects ( $\mu$ invalid $-\mu$ valid) as well as the number of missed responses between the placebo and nicotine group (in case of the validity effects a one-tailed significance was used due to our directed hypothesis of a reduced validity effect with nicotine). Moreover, we correlated the validity effect as well as the parameters $\mu, \sigma$, and $\tau$ with blood nicotine level scores using Pearson's correlation coefficient.

\section{Eye Movement Control}

Eye position was monitored during scanning with an MRcompatible infrared eye tracker (ASL Model 540, Applied Science Group Co., Bedford, MA). Eye data were analyzed with ILAB software (Gitelman, 2002). Artefacts related to blinking were filtered out. A region of interest subtending $25 \%$ of the cue-target distance from the center was defined as fixation zone. We calculated for each subject and cue validity condition the amount of time spent in this central region between cue and target.

\section{RESULTS}

\section{Physiological and Subjective Measures}

Nicotine significantly increased the pulse rate (placebo group: $68.8 \pm 2.7$ mean $\pm S E M$, nicotine group: $78.2 \pm 2.6$, $\mathrm{t}(22)=-2.5, \quad P<0.05)$. Nicotine blood levels could be determined in 11 of the 12 subjects of the nicotine group and amounted to $3.57 \pm 0.4 \mathrm{ng} / \mathrm{ml}$. The data of one sample was lost due to technical problems. The result is comparable with a prior study on the effect of nicotine chewing gums on blood nicotine levels in healthy non-smokers using gums containing $4 \mathrm{mg}$ of nicotine (ie, twice as much as in the present study). Here, blood nicotine levels amounted on average to $6.53 \mathrm{ng} / \mathrm{ml}$ after $30 \mathrm{~min}$ of chewing (Nyberg et al, 1982).

There were no significant differences between the two groups with respect to their subjective ratings of alertness, contentedness, and calmness. None of the subjects reported 
task-interfering side effects of the chewing gums (like, eg, manifest feelings of nausea or dizziness).

\section{Eye Movement Data}

Eye movement data were reliably recordable in 14 subjects (six subjects of the placebo group and eight subjects of the nicotine group) only. Analysis of this data showed that there were no significant differences in fixation performance between the 90 and the $60 \%$ cue validity condition and the drug and placebo group, respectively (placebo group: $96.6 \pm 1.8$ and $96.8 \pm 1.9 \%$; nicotine group: $93.3 \pm 4.5$ and $93.6 \pm 4.3 \%$; all $P>0.5$ ). Note that in all subjects fixation was additionally monitored by visual inspection of the participants' eyes during scanning.

\section{Behavioral data}

Missed responses, anticipations, and false alarms (catch trials) amounted to $4.3,0.7$, and $6.2 \%$ in the placebo and $1.9,0.5$, and $5.6 \%$ in the nicotine group, respectively. The difference in missed responses between the two groups was not significant $(P<0.087$, all other $P>0.4)$.

The effects of cue validity ( $90 v s 60 \%$ ), cueing (valid $v s$ invalid), and drug (placebo $v s$ nicotine) were tested with ANOVAs on the parameters of the ex-Gauss RT distribution. Table 1 depicts the parameter $\mu$ (ie, the mean of the Gaussian component) for each group and stimulus condition. We found a significant main effect of cueing $(\mathrm{F}(1,22)=64.49 ; P<0.001)$ reflecting faster $\mathrm{RT}$ in valid as compared with invalid trials (ie, the validity effect). The ANOVA further yielded a cue validity $\times$ drug interaction $(\mathrm{F}(1,22)=16.09 ; \quad P<0.001)$ and a cueing $\times$ cue validity interaction $(\mathrm{F}(1,22)=6.07 ; \quad P<0.05)$. Importantly, the three-way interaction of cue validity $\times$ cueing $\times$ drug was significant $(\mathrm{F}(1,22)=10.81 ; P<0.01)$ reflecting a differential nicotinic modulation of RTs in invalid trials in the 90 and $60 \%$ cue validity condition, respectively. Figure 1 illustrates this interaction in showing that nicotine specifically modulated the validity effect in the $90 \%$ cue validity condition $(\mathrm{t}(22)=1.85 ; P<0.05)$.

For the parameter $\sigma$ (ie, the standard deviation of the Gaussian component of the RT distribution), we observed a significant main effect of cueing $(\mathrm{F}(1,22)=12.88 ; P<0.01)$ as well as cue validity $\times \operatorname{drug}(\mathrm{F}(1,22)=20.3 ; P<0.001)$ and cueing $\times$ cue validity $\times$ drug interactions $(\mathrm{F}(1,22)=14.04$; $P<0.001)$. This finding reflects that nicotine reduced RT variability in invalid trials in the context of $90 \%$ cue validity, which is illustrated in Figure 2. The ANOVA of the exponential component of the ex-Gauss function $\tau$ yielded significant cue validity $\times \operatorname{drug}(\mathrm{F}(1,22)=14.38 ; P<0.001)$ and cueing $\times$ cue validity $\times$ drug $(\mathrm{F}(1,22)=12.88 ; P<0.01)$ interactions. This result reflects a steeper decline in the

Table I Behavioral Data. Mean of the Gaussian Component $(\mu)$ of the Ex-Gauss Distributions ( \pm SEM)

\begin{tabular}{lcccc}
\hline & Valid 90\% & Invalid 90\% & Valid 60\% & Invalid 60\% \\
\hline Placebo & $233.0 \pm 6.7$ & $308.0 \pm 15.9$ & $236.9 \pm 7.2$ & $277.1 \pm 13.3$ \\
Nicotine & $241.2 \pm 6.6$ & $289.1 \pm 13.0$ & $246.2 \pm 10.1$ & $299.1 \pm 14.2$ \\
\hline
\end{tabular}

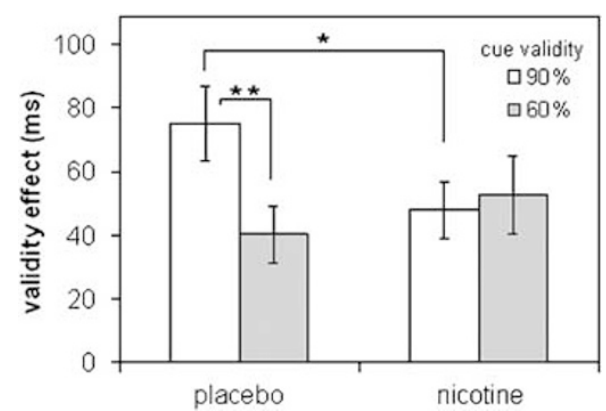

Figure I Behavioral data. Validity effects for the two cue validity conditions in the placebo and nicotine group. $* P<0.05$; ${ }^{*} * P<0.00$ I.

probability function in invalid trials in the $60 \%$ cue validity condition under nicotine (Figure 2) indicating fewer outlying slow RT values.

No significant correlations between the size of the validity effect and blood nicotine level were observed. Interestingly, however, blood nicotine levels correlated negatively with the parameter $\sigma$ in invalid trials in the $90 \%$ cue validity condition ( $r=-0.68 ; P<0.05$, two-tailed significance) suggesting an association between increases in nicotine and the consistency of responding in this experimental condition.

\section{Neural Data}

We identified those brain regions, which showed stronger reductions of reorienting-related neural activity (invalid $>$ valid) in the $90 \%$ than in the $60 \%$ cue validity condition in response to nicotine by a directed $t$-contrast capturing the interaction effect of the factors cue validity, cueing, and drug. This contrast revealed activation in temporo-parietal and parietal brain areas of the right hemisphere. The averaged parameter estimates (beta weights) for activations in these brain regions are plotted in Figure 3 to illustrate BOLD signal changes in these brain areas as a function of drug, cueing, and cue validity.

Note that the increased BOLD signal in invalid trials in the $90 \%$ cue validity condition under placebo and its reduction under nicotine mirrors the behavioral data and is particularly evident near the right temporo-parietal junction (TPJ) and in the right superior parietal gyrus adjacent to the intraparietal sulcus. Interestingly, neural activity in invalid trials in the $60 \%$ cue validity condition showed the reversed pattern in the right angular and superior parietal gyrus with higher activity in the nicotine than in the placebo group. In addition, the interaction contrast yielded activation in right frontal brain regions as well as in a region in the left anterior cingulate cortex (Figure 3d). A complete list of all activations obtained is provided in Table 2. Significant correlations of neural activity with blood nicotine levels were observed for two brain regions (right precentral gryus and left middle temporal gyrus) only. Here, the activity in invalid trials in the context of high cue validity correlated negatively with the blood levels of nicotine (precentral gyrus: $r=-0.73, P<0.01$; middle temporal gyrus: $r=-0.69$, $P<0.05)$. The latter correlation, however, was mainly caused by few outlying values.

The reverse contrast yielded one activation cluster in the left postcentral gyrus $(x=-57, y=-18, z=30$; $Z=3.57,3$ voxels). 

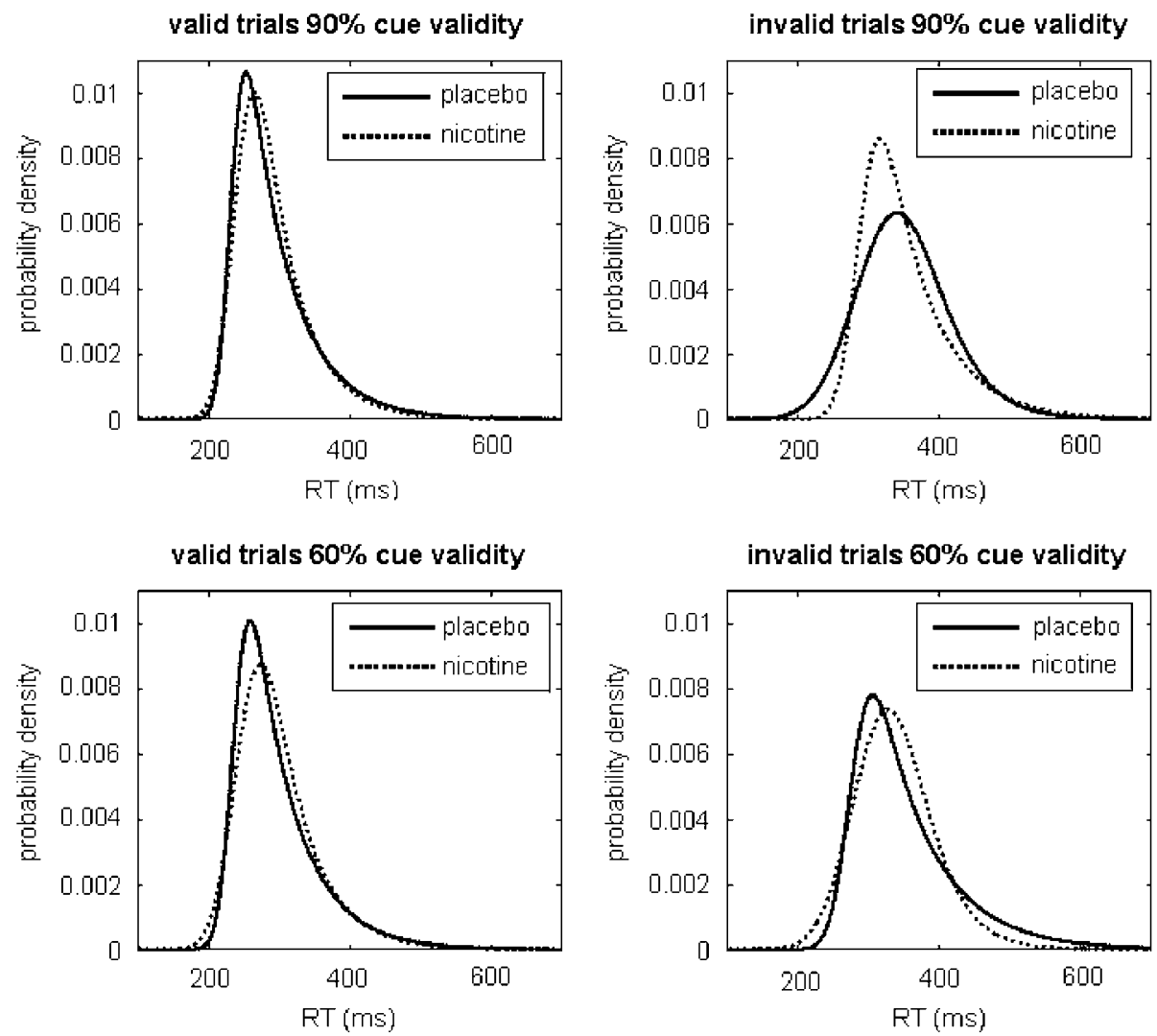

Figure 2 Behavioral data. Probability density functions for the four experimental conditions in the placebo and nicotine group.

\section{DISCUSSION}

Using the combination of fMRI and psychopharmacology, we show that attentional reorienting in the location-cueing task is influenced by the cholinergic agonist nicotine specifically in situations of high cue validity. Brain areas contributing to this effect are right fronto-parietal as well as left anterior cingulate regions.

\section{Behavioral Data}

The behavioral data demonstrate a decrease in the validity effect in the $90 \%$ cue validity condition after nicotine administration. The significant three-way interaction of the factors cueing, cue validity, and drug suggests that a cholinergic facilitation of attentional reorienting is observed in situations of high cue validity only. Note, that for the $60 \%$ cue validity condition, the magnitude of the validity effect was numerically even higher under nicotine. Furthermore, nicotine reduced the variability of RTs to invalidly cued targets in the context of high cue validity and this effect correlated with serum nicotine levels. Moreover, we observed a tendency towards fewer missed responses under nicotine.

Decreased RT variability and omission rates in response to nicotine have also been reported in a study using the Continuous Performance Test (CPT, Conners, 1995), which assesses sustained attention abilities (Levin et al, 1998). Although sustained attention is required for successful performance in location-cueing paradigms and presumably affects RT variability and missed responses, our results cannot solely be explained by increases in sustained attention. In particular, we did not observe a general speeding of RTs in the nicotine group. RTs in valid trials in the 90 and $60 \%$ cue validity condition as well as RTs in invalid trials in the low cue validity condition were numerically even higher in the group receiving nicotine (Table 1). However, as invalid trials occur per definition less frequently in a high than in a low cue validity condition, one could still argue that the observed nicotinic effect depends on stimulus frequency rather than on reorienting processes. Evidence against a dependence of the effect of nicotine on stimulus frequency is provided by a behavioral study (Meinke, 2006), which demonstrated that a low 'go probability' condition (in which a response is required in a small proportion of trials only, ie, to infrequent target stimuli) is not sufficient to evoke a nicotinic reduction of the validity effect. Our results therefore argue for a specific modulatory effect of nicotine on attentional reorienting (Witte et al, 1997; Murphy and Klein, 1998), which strongly depends on the a priori validity of the spatial cues.

We have previously suggested (Thiel et al, 2005) that the reduction of the validity effect under nicotine depends on the size of the validity effect under placebo. In the present study, we manipulated the size of the validity effect experimentally by employing different cue validities. Using such a within-subject manipulation, we were able to show that nicotine acts specifically in situations in which high validity effects are present. What determines the size of the validity effect? Attentional gradient models assume that processing resources are allocated according to the spatial cues and that this resource allocation is influenced by the 


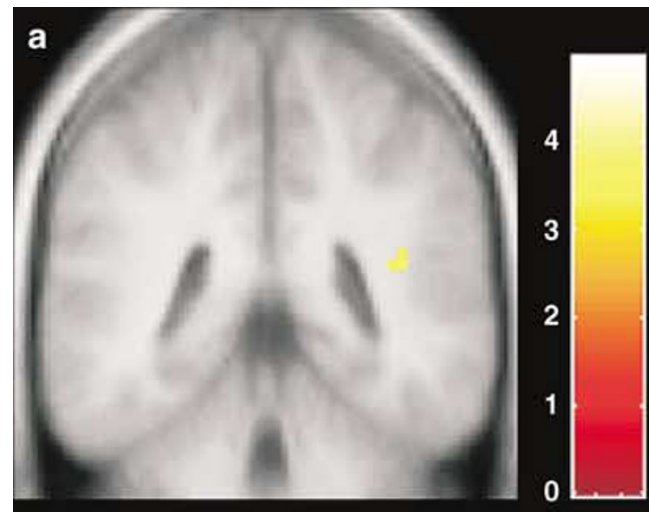

[42 -42 15]
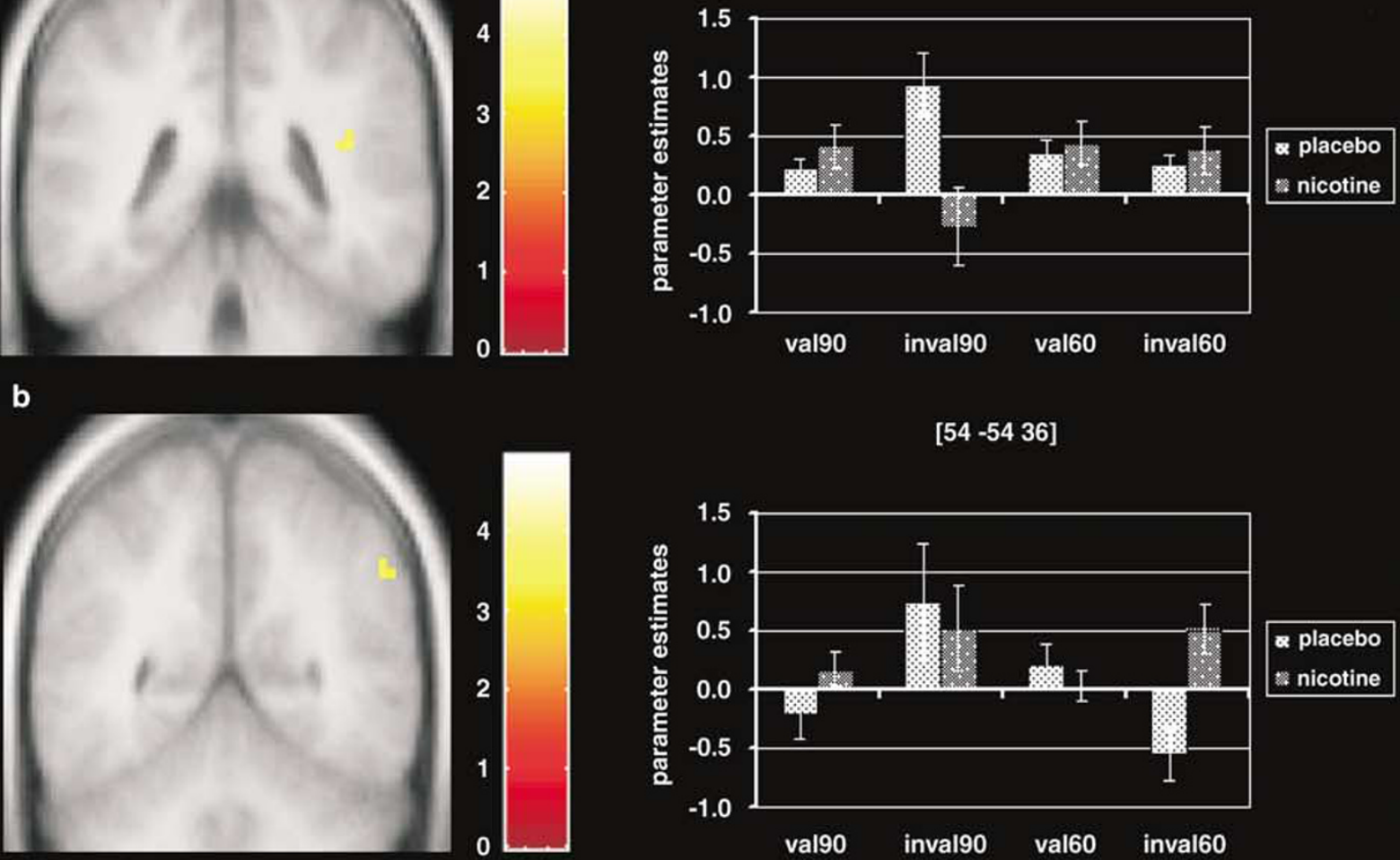

c

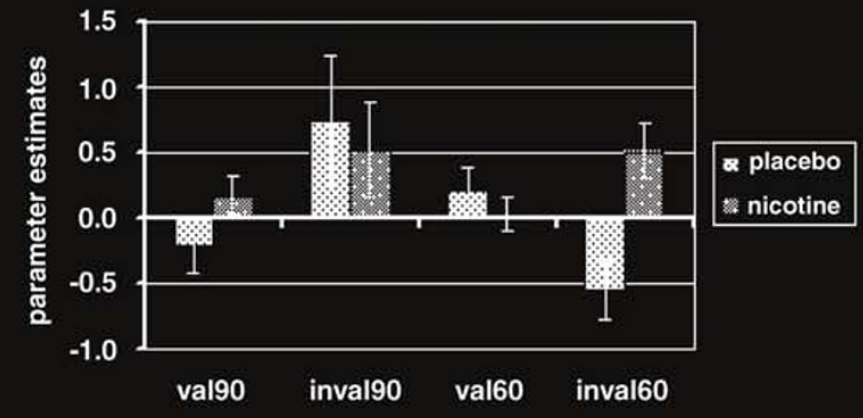

[18 -60 63]
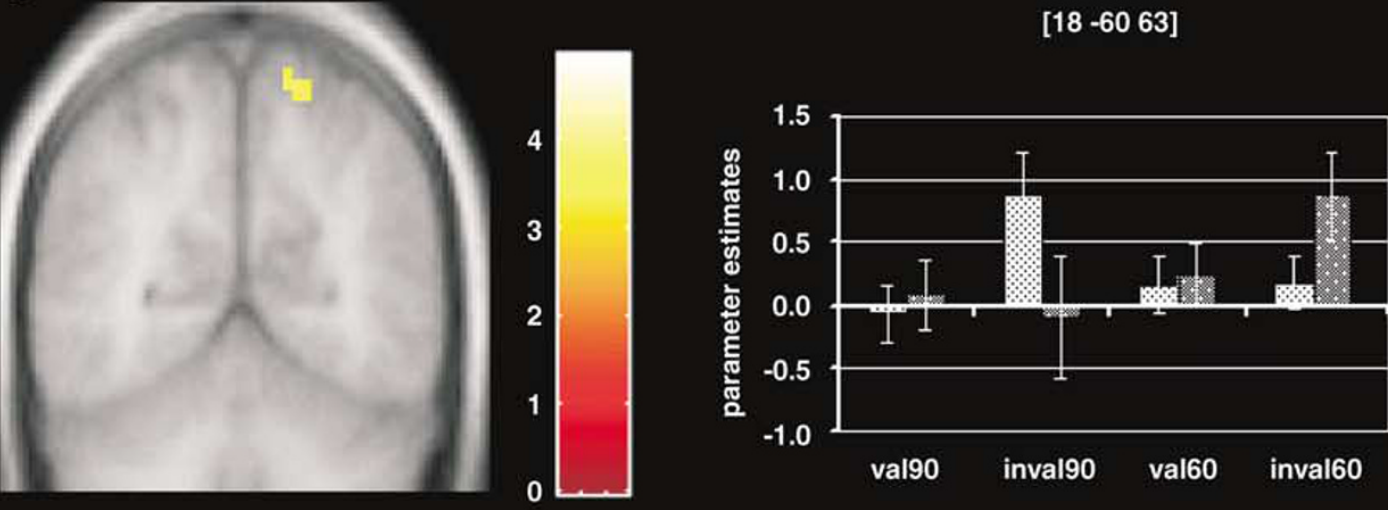

\& placebo

a nicotine

d

$\left[\begin{array}{lll}-15 & 30 & -6\end{array}\right]$
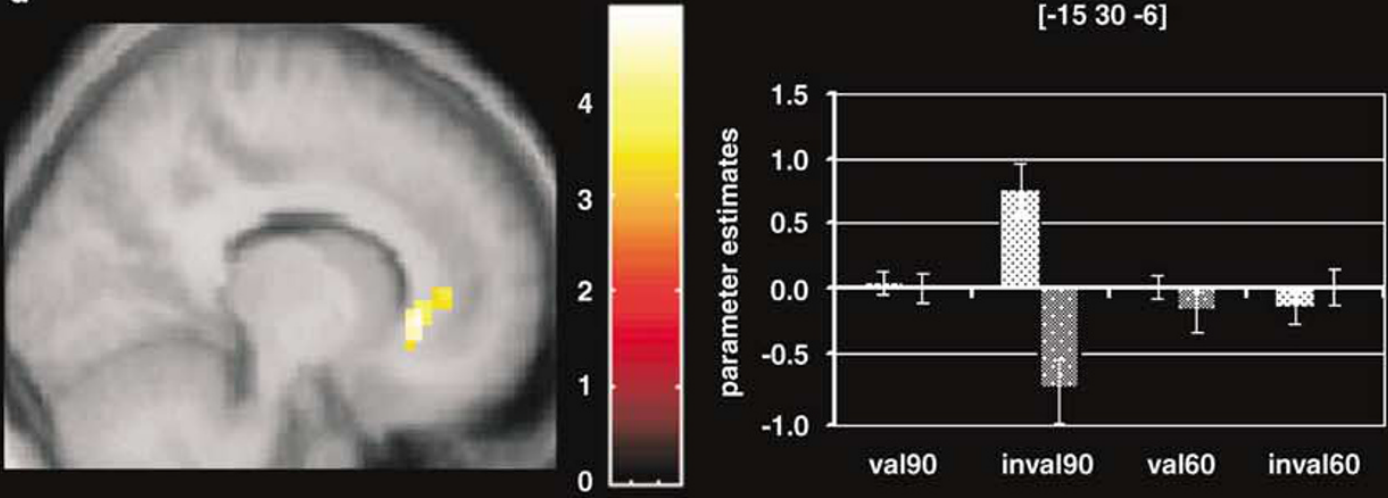

o placebo

w nicotine

Figure 3 Coronal $(\mathrm{a}-\mathrm{c}$ ) and sagittal (d) sections through parietal, temporo-parietal, and cingulate brain regions showing cueing $\times$ cue validity $\times$ drug interaction effects. (a) Right superior temporal gyrus/TPJ (b) right angular gyrus (c) right superior parietal gyrus/IPS (d) left anterior cingulate gyrus. val90: valid trials $90 \%$ cue validity; inval90: invalid trials in the context of $90 \%$ cue validity; val60: valid trials $60 \%$ cue validity; inval 60 : invalid trials in the context of $60 \%$ cue validity. 
Table 2 Neural Data. Cortical Regions Showing Stronger Reductions in Reorienting-Related Neural Activity (Invalid > Valid) in the $90 \%$ than in the $60 \%$ Cue Validity Conditions in Response to Nicotine

\begin{tabular}{|c|c|c|c|c|c|c|}
\hline \multirow[b]{2}{*}{ Region } & \multirow[b]{2}{*}{ Side } & \multicolumn{3}{|c|}{ MNI coordinates } & \multirow[b]{2}{*}{ Voxels } & \multirow[b]{2}{*}{$Z$ score } \\
\hline & & $x$ & $y$ & $\mathbf{z}$ & & \\
\hline \multicolumn{7}{|l|}{ Frontal } \\
\hline Superior orbital gyrus & $\mathrm{R}$ & 15 & 54 & -15 & 8 & 3.98 \\
\hline \multirow[t]{2}{*}{ Middle frontal gyrus } & $\mathrm{R}$ & 51 & 15 & 45 & 5 & 3.67 \\
\hline & $\mathrm{R}$ & 36 & 60 & 3 & 6 & 3.39 \\
\hline Precentral gyrus & $\mathrm{R}$ & 51 & 0 & 24 & 4 & 3.65 \\
\hline \multicolumn{7}{|l|}{ Temporal } \\
\hline Middle temporal gyrus & $L$ & -51 & -18 & -21 & 3 & 3.25 \\
\hline $\begin{array}{l}\text { Superior temporal } \\
\text { gyrus/TPJ }\end{array}$ & $\mathrm{R}$ & 42 & -42 & 15 & 3 & 3.60 \\
\hline \multicolumn{7}{|l|}{ Parietal } \\
\hline Superior parietal cortex & $\mathrm{R}$ & 18 & -60 & 63 & 8 & 3.45 \\
\hline Angular gyrus & $\mathrm{R}$ & 54 & -54 & 36 & 4 & 3.24 \\
\hline \multicolumn{7}{|l|}{ Occipital } \\
\hline Superior lingual gyrus & $\mathrm{R}$ & 24 & -48 & 3 & 6 & 3.57 \\
\hline \multicolumn{7}{|l|}{ Other } \\
\hline Anterior cingulate gyrus & L & -15 & 30 & -6 & 30 & 4.53 \\
\hline Hippocampus & $\mathrm{R}$ & 33 & -12 & -18 & 6 & 3.35 \\
\hline White matter & $\mathrm{R}$ & 30 & -27 & 3 & 4 & 3.54 \\
\hline Corpus callosum & $\mathrm{R}$ & 3 & 9 & 15 & 9 & 3.53 \\
\hline
\end{tabular}

validity of the cues (Madden, 1992). In a high cue validity condition, the resource distribution has a strong peak at the cued location with only few resources at the uncued location leading to high validity effects. Conversely, the resource distribution in a low cue validity condition exhibits a lower maximum at the cued but more available resources at the uncued location, decreasing the validity effect. The data presented here suggest that nicotine promotes cue uncertainty ( $\mathrm{Yu}$ and Dayan, 2005), and redistributes the attentional resources in favor of the uncued location in the high cue validity condition. This results in speeded target detection in invalid trials at the cost of target detection in validly cued trials. Thus, the current data provide further evidence that nicotine flattens the distribution of attentional resources in situations where the resource distribution has a strong peak due to top-down modulation.

\section{Neural Data}

The present study aimed at identifying those brain areas related to attentional reorienting in which neural activity is differentially modulated as a function of cue validity and cortical acetylcholine levels (nicotinic cholinergic stimulation $v s$ placebo). In accordance with our hypothesis, we observed a nicotine-induced reduction of reorientingrelated activity in right parietal brain regions (superior parietal cortex/IPS and TPJ) in invalid trials in the context of high cue validity. Both cortical regions are known to play key roles in attentional processes. The IPS is part of an orienting network responsible for the voluntary control of attention, that is, for the top-down selection of stimuli (Corbetta and Shulman, 2002). The TPJ region on the other hand is supposed to signal the appearance of unexpected or unattended events to this orienting network (Corbetta and Shulman, 2002) and it has been suggested that this temporo-parietal area is involved in balancing top-down information and bottom-up sensory input (Serences et al, 2005). Thus, the reduced activity of the TPJ and the IPS region under nicotine could be the neural mechanism underlying the behavioral nicotinic effect of a reduced impact of top-down expectations.

Interestingly, while reducing neural activity in invalid trials in the $90 \%$ cue validity condition, nicotine increased activity in invalid trials in the $60 \%$ cue validity condition in parietal brain areas (superior parietal cortex/IPS, angular gyrus). These results might seem inconsistent with the findings of Giessing et al (2006) showing reduced activity in areas adjacent to the intraparietal sulcus in invalid trials in the context of $64 \%$ cue validity. One possible reason for these discrepancies could be the use of two different cues in the present study. Whereas the study by Giessing et al (2006) employed one cue type only (ie, cue validity was manipulated across different experimental blocks), the task in this study required a matching of cue identity (green, blue) and cue validity (90,60\%). This could have emphasized the differences between the two experimental conditions, thereby possibly promoting the observed dissociation in the neuromodulatory effects of nicotine in the two cue validity conditions.

How could the pharmacological modulation of parietal brain regions be accomplished? Sarter et al (2005) proposed that the cortical cholinergic fibers emanating from the basal forebrain project to an 'anterior attention system', which regulates the activity of posterior brain areas and includes the prefrontal cortex. The present study yielded a cueing $\times$ cue validity $\times$ drug interaction in the middle frontal gyrus within the right prefrontal cortex and within the left anterior cingulate cortex. It has been suggested that the anterior cingulate and prefrontal cortex are part of a system involved in effortful cognitive control, which is supposed to be mediated by cholinergic neurotransmission (Sarter et al, 2006). However, the question whether the anterior cingulate cortex is only involved in the detection of negative events (like, eg, errors) or rather represents an instance of control itself remains a matter of debate (Sarter et al, 2006). Thus, the activation observed could, on the one hand, be linked to the processing of attentional effort related to reorienting attention in the two different cue validity conditions. On the other hand, however, we observed higher response variability in invalid trials in the high cue validity condition under placebo. Hence, provided that the subjects appraised these occasional delays in responding in this experimental condition as deficient performance in the task, the activation of the anterior 
cingulate cortex could as well be attributed to error detection processes (eg, Menon et al, 2001).

Taken together, our results suggest interaction effects of the pharmacological and cognitive modulations of attentional reorienting, which can be observed on both a behavioral and a neuronal level.

\section{ACKNOWLEDGEMENTS}

We are grateful to our colleagues from the MR and Cognitive Neurology groups for valuable support and discussions.

\section{DISCLOSURE/CONFLICT OF INTEREST}

We declare that, except for income received from the primary employer and grants from the Deutsche Forschungsgemeinschaft (KFO-112, TP 8) to CMT and GRF, no financial support or compensation has been received from any individual or corporate entity over the past 3 years for research or professional service and there are no personal financial holdings that could be perceived as constituting a potential conflict of interest. The placebo chewing gums used in the present study were provided by courtesy of Pharmacia/Pfizer (Helsingborg, Sweden).

\section{REFERENCES}

Bond A, Lader M (1974). The use of analogue scales in rating subjective feelings. Br J Clin Pharmacol 47: 211-218.

Bowman EM, Brown VJ, Kertzman C, Schwarz U, Robinson DL (1993). Covert orienting of attention in macaques: I. Effects of behavioural context. J Neurophysiol 70: 431-443.

Conners CK (1995). The Continuous Performance Test. MultiHealth Systems: Toronto.

Corbetta M, Shulman GL (2002). Control of goal-directed and stimulus-driven attention in the brain. Nat Rev Neurosci 3: 201-215.

Eriksen CW, Yeh Y-Y (1985). Allocation of attention in the visual field. J Exp Psychol Hum Percept Perform 11: 583-597.

Friston KJ, Holmes AP, Worsley KJ, Poline J-P, Frith CD, Frackowiak RS (1995). Statistical parametric maps in functional imaging: a general linear approach. Hum Brain Mapp 2: 189-210.

Giessing C, Thiel CM, Rösler F, Fink GR (2006). The modulatory effects of nicotine on parietal cortex depend on cue reliability. Neuroscience 137: 853-864.

Gitelman DR (2002). ILAB: a program for postexperimental eye movement analysis. Behav Res Meth Instrum Comput 34: 605-612.

Gottlob L (2004). Location cuing and response time distributions in visual attention. Perception \& Psychophysics 66: 1293-1302.

Griesar WS, Zajdel DP, Oken BS (2002). Nicotine effects on alertness and spatial attention in non-smokers. Nicotine Tob Res 4: 185-194.

Heathcote A, Brown S, Cousineau D (2004). QMPE: estimating lognormal, wald and weibull RT distributions with a parameter dependent lower bound. Behav Res Meth Instrum Comput 36: 277-290.

Heathcote A, Popiel SJ, Mewhort DJK (1991). Analysis of response time distributions: an example using the stroop task. Psychol Bull 109: 340-347.

Hills M, Armitage P (1979). The two-period cross-over clinical trial. Br J Clin Pharmacol 8: 7-20.

Jonides J (1980). Towards a model of the mind's eye's movement. Can J Psychol 34: 103-112.
Josephs O, Henson RN (1999). Event-related functional magnetic resonance imaging: modelling: inference and optimization. Philos Trans R Soc Lond B Biol Sci 354: 1215-1228.

Levin ED, Conners CK, Silva D, Hinton SC, Meck WH, March J et al (1998). Transdermal nicotine effects on attention. Psychopharmacology 140: 135-141.

Madden DJ (1992). Selective Attention and visual search: revision of an allocation model and application to age differences. $J$ Exp Psychol Hum Percept Perform 18: 821-836.

Meinke A (2006). Nikotineffekte auf räumliche Aufmerksamkeitsprozesse bei Nichtrauchern (PhD thesis, University of Potsdam, Germany. URL: http://opus.kobv.de/ubp/volltexte/2006/765/).

Meinke A, Thiel CM, Fink GR (2006). Effects of nicotine on visuospatial selective attention as indexed by event-related potentials. Neuroscience 141: 201-212.

Menon V, Adleman NE, White CD, Glover GH, Reiss AL (2001). Localization of a neural system for error detection. Hum Brain Mapp 12: 131-143.

Millar K (1983). Clinical trial design: the neglected problem of asymmetrical transfer in cross-over trials. Psychol Med 13: 867-873.

Murphy FC, Klein RM (1998). The effects of nicotine on spatial and non-spatial expectancies in a covert orienting task. Neuropsychologia 36: 1103-1114.

Newhouse PA, Potter A, Singh A (2004). Effects of nicotinic stimulation on cognitive performance. Curr Op Pharmacol 4: 36-46.

Nyberg G, Panfilov V, Sivertsson R, Wilhelmsen L (1982). Cardiovascular effects of nicotine chewing gum in healthy non-smokers. Eur J Clin Pharmacol 23: 303-307.

Oldfield RC (1971). The assessment and analysis of handedness: the Edinburgh Inventory. Neuropsychologia 9: 97-113.

Phillips JM, McAlonan K, Robb WG, Brown VJ (2000). Cholinergic neurotransmission influences covert orienting of visuospatial attention in the rat. Psychopharmacology 150: 112-116.

Posner MI (1980). Orienting of attention. Q J Exp Psychol 32: 3-25.

Posner MI, Fan J (2004). Attention as an organ system. Neurobiology of Perception and Communication: from Synapse to Society. The IVth De Lange Conference. Cambridge University Press: Cambridge.

Rezvani AH, Levin ED (2001). Cognitive effects of nicotine. Biol Psychiatry 49: 258-267.

Riggio L, Kirsner K (1997). The relationship between central and peripheral cues in covert visual attention. Percept Psychophys 59: 885-899.

Sarter M, Gehring WJ, Kozak R (2006). More attention must be paid: the neurobiology of attentional effort. Brain Res Brain Res Rev 51: 145-160.

Sarter M, Hasselmo ME, Bruno JP, Givens B (2005). Unraveling the attentional functions of cortical cholinergic inputs: interactions between signal-driven and cognitive modulation of signal detection. Brain Res Brain Res Rev 48: 90-111.

Serences JT, Shomstein S, Leber AB, Golay X, Egeth HE, Yantis S (2005). Coordination of voluntary and stimulus-driven attentional control in human cortex. Psychol Sci 16: 114-122.

Stewart C, Burke S, Marrocco R (2001). Cholinergic modulation of covert attention in the rat. Psychopharmacology 155: 210-218.

Thiel CM, Zilles K, Fink GR (2005). Nicotine modulates reorienting of visuospatial attention and neural activity in human parietal cortex. Neuropsychopharmacology 30: 810-820.

Vossel S, Thiel CM, Fink GR (2006). Cue validity modulates the neural correlates of covert endogenous orienting of attention in parietal and frontal cortex. Neuroimage 32: 1257-1264.

Witte EA, Davidson MC, Marrocco RT (1997). Effects of altering brain cholinergic activity on covert orienting of attention: comparison of monkey and human performance. Psychopharmacology 132: 324-334.

Yu AJ, Dayan P (2005). Uncertainty, neuromodulation, and attention. Neuron 46: 681-692. 